Research Article

\title{
Simultaneous Quantification of Aspirin, Its Metabolite Salicylic Acid, and Salvianolic Acid B in Human Plasma Using UPLC-MS/MS
}

\author{
Weiyi Cao ${ }^{1 D},{ }^{1,2}$ Hezhi Yao, ${ }^{1}$ Zhenzhen Qian, ${ }^{1}$ Yi Wu, ${ }^{1,2}$ Shuge Wang, ${ }^{1,2}$ Rui Li $\left(\mathbb{D},{ }^{1,2}\right.$ \\ and Rui Gao iD ${ }^{1,2}$ \\ ${ }^{1}$ Institute of Clinical Pharmacology of Xiyuan Hospital, China Academy of Chinese Medical Sciences, No. 1, R. Xiyuangcaochang, \\ Haidian District, Beijing 100091, China \\ ${ }^{2}$ NMPA Key Laboratory for Clinical Research and Evaluation of Traditional Chinese Medicine, \\ National Clinical Research Center for Chinese Medicine Cardiology, Beijing, China
}

Correspondence should be addressed to Rui Li; crystal005@163.com and Rui Gao; xyyygr@126.com

Received 24 November 2020; Revised 7 February 2021; Accepted 15 March 2021; Published 29 March 2021

Academic Editor: David Touboul

Copyright (c) 2021 Weiyi Cao et al. This is an open access article distributed under the Creative Commons Attribution License, which permits unrestricted use, distribution, and reproduction in any medium, provided the original work is properly cited.

Salvianolic acid B is the main active ingredient in salvianolate injection, which is produced by extracting danshen, the most commonly used Chinese herbal medicine for cardiovascular treatment. Clinically, salvianolate injection and aspirin are commonly combined to treat coronary heart diseases in patients with stable angina. To support clinical studies on drug-drug interactions (DDIs) between salvianolate injection and aspirin, a rapid and sensitive UPLC-MS/MS method for the simultaneous determination of aspirin (acetylsalicylic acid), its metabolite salicylic acid, and salvianolic acid B in human plasma was developed. The analytes and internal standard were extracted from the acidified plasma by liquid-liquid extraction with ethyl acetate and then separated by gradient elution with acetonitrile/ $0.5 \%$ formic acid in water on a C18 column. Salvianolic acid B, acetylsalicylic acid, and salicylic acid were quantified in multiple-reaction monitoring mode with negative ion electrospray ionization. The method was fully validated according to the current regulatory guidance for bioanalysis. Calibration curves in the range $5-6000 \mathrm{ng} / \mathrm{mL}$, all with correlation coefficients greater than 0.99 , were established using linear regression models for salvianolic acid B and acetylsalicylic acid and a quadratic model for salicylic acid. The validated method was successfully used to measure salvianolic acid B, acetylsalicylic acid, and salicylic acid concentrations in human plasma samples from 16 patients to observe the pharmacokinetic changes caused by DDIs.

\section{Introduction}

Salvianolate injection, which is prepared by extracting danshen, the most widely used Chinese medicinal herb, is used clinically to treat coronary heart disease in patients with stable angina [1]. A systematic review and meta-analysis have revealed that salvianolate injection in combination with Western medicine could improve the total therapeutic rate and electrocardiogram effective rate [2] as well as reduce the hospitalization time of patients with coronary heart disease [3]. Pharmacological studies have indicated that salvianolic injection could protect cardiomyocytes by inhibiting TGF- $\beta 1 / \mathrm{Smad} 2 / 3$ and TXNIP/ NLRP3 inflammatory signaling pathways [4]. Furthermore, salvianolic acid B (Sal B), the major component of salvianolate injection, could inhibit human platelet activation via PDE inhibition and P2Y12 antagonism [5]. In addition, Sal B could reduce the inflammatory reaction, even if the platelets have already been activated [6].

Clinically, salvianolate injection is often used in combination with aspirin (acetylsalicylic acid; ASA) to treat cardiovascular disease. Clinical research has shown that danshen preparations combined with ASA significantly relieve the symptoms of angina pectoris [7] and reduce the damage to the gastric mucosa caused by ASA and the risk of gastric bleeding [8]. Furthermore, laboratory studies have revealed a potential pharmacokinetic interaction between danshen and ASA $[9,10]$. 
In particular, the combined use of salvianolate injection and ASA may improve the efficacy and reduce the toxicity by changing the pharmacokinetic behavior. Therefore, clinical research on the drug-drug interactions (DDIs) between salvianolate injection and ASA is necessary to guide the rational clinical use of this medication.

To conduct pharmacokinetic research on DDIs between salvianolate injection and ASA, a simple and sensitive detection method is required. In biological samples, ASA and its metabolite salicylic acid (SA) are often quantified together with other cardiovascular drugs. A reported combined detection method for aspirin and clopidogrel uses protein precipitation combined with liquid-liquid extraction to process plasma samples; however, the complexity of these operations may increase the experimental error $[10,11]$. In addition, liquid-liquid extraction using methyl tert-butyl ether has been used in combined detection methods for aspirin with pravastatin [12] or with atorvastatin [13]. For acidification before liquid-liquid extraction, a relatively mild organic acid, such as $1 \%$ formic acid (FA), is typically used to reduce the ionization of ASA and SA in plasma [12]. However, Sal B usually requires a strong acid such as hydrochloric acid as an acidifying agent [14]. In addition, when redissolving the extraction residue, acetonitrile (ACN) [11] or a mixed solution with a high proportion of $\mathrm{ACN}$, such as $\mathrm{ACN} / \mathrm{H}_{2} \mathrm{O}(80: 20, \mathrm{v} / \mathrm{v}$, containing $0.5 \%$ acetic acid $)$ [10] or $\mathrm{ACN} / \mathrm{H}_{2} \mathrm{O}(80: 20, \mathrm{v} / \mathrm{v}$, containing $5 \mathrm{mM}$ ammonium acetate buffer) [12], is typically used for ASA and SA. In contrast, a solvent mixture that contains methanol $(\mathrm{MeOH})$, such as $\mathrm{MeOH} / \mathrm{H}_{2} \mathrm{O}(50: 50, \mathrm{v} / \mathrm{v})$, is necessary for Sal B [15]. Therefore, none of the currently available biological sample analysis methods are suitable for the simultaneous detection of ASA, SA, and Sal B. Chemical properties should also be considered when choosing an internal standard. Chloramphenicol, which is an acidic compound with a low baseline in the plasma matrix, has been reported as an internal standard for the in vivo analysis of Sal B $[16,17]$. Moreover, in view of the inherent problems associated with the bioanalysis of Sal B, ASA, and SA, including a lack of stability and high polarity [18], establishing a suitable bioanalytical method is challenging. LC-MS/MS is considered a gold standard for biological analysis with high throughput, sensitivity, specificity, small sample volume requirements, and suitability for analyzing large numbers of clinical samples. However, to date, no bioanalytical UPLC-MS/MS method has been reported for the simultaneous analysis of Sal B, ASA, and SA.

In this work, we developed a UPLC-MS/MS method for the simultaneous determination of Sal B, ASA, and SA in human plasma. In addition, the applicability of the validated method to a clinical pharmacokinetic study of the changes caused by DDIs between salvianolate injection and ASA was investigated.

\section{Materials and Methods}

2.1. Chemicals and Materials. Sal B (lot P10J9F52565, purity $>98 \%$ ), ASA (lot SJ0714YA14, purity > 98\%), SA (lot J22S6J3607, purity $>98 \%$ ), and chloramphenicol (IS; lot HM0328NA14, purity >97\%) were purchased from Shanghai Yuanye Bio-Technology Co., Ltd. (Shanghai,
China). The chemical structures of Sal B, ASA, SA, and IS are shown in Figure 1.

HPLC-grade ACN, HPLC-grade $\mathrm{MeOH}$, and Optima LC/ MS-grade FA were purchased from Fisher Scientific, Inc. (Fair Lawn, NJ, USA). High-purity water was obtained using a MilliQ Ultrapure Water purification system (Millipore, Burlington, MA, USA). Blank plasma samples were obtained from healthy volunteers with EDTA used as an anticoagulant.

Salvianolate injection (lot 17090623, $200 \mathrm{mg}+5 \%$ glucose injection, in $250 \mathrm{~mL}$, iv) was purchased from Shanghai Green Valley Pharmaceutical Co., Ltd. (Shanghai, China). Aspirin enteric-coated tablets (lot BJ38708, $100 \mathrm{mg}$ ) were purchased from Bayer Healthcare Co., Ltd. (Beijing, China).

2.2. UPLC-MS/MS Conditions. A UPLC system (consisting of an Acquity Binary Solvent Manager and an Acquity Sample Manager, Waters, Milford, MA, USA) coupled with

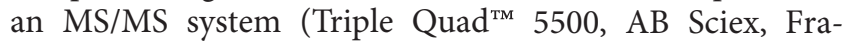
mingham, MA, USA) was applied for the determination of ASA, SA, and Sal B in biosamples. The data were collected and processed using the Analyst software (version 1.6.2, AB Sciex, Framingham, MA, USA).

Chromatographic separation was achieved using an Acquity UPLC ${ }^{\text {тм }}$ BEH C18 column $(2.1 \times 100 \mathrm{~mm}, 1.7 \mu \mathrm{m}$, Waters, Wexford, Ireland) at $50^{\circ} \mathrm{C}$. The mobile phase consisted of $0.5 \% \mathrm{FA}$ in water (solvent $\mathrm{A}$ ) and $\mathrm{ACN}$ (solvent $\mathrm{B}$ ). The gradient elution program was as follows: $0-0.3 \mathrm{~min}$, 95-75\% A; 0.3-1.3 min, 75-70\% A; 1.3-2.1 min, 70-30\% A; 2.1-2.5 min, $30 \% \quad \mathrm{~A} ; \quad 2.5-2.7 \mathrm{~min}, \quad 30-95 \% \mathrm{~A}$; and 2.7-4.0 $\mathrm{min}, 95 \% \mathrm{~A}$. The flow rate was $0.4 \mathrm{~mL} / \mathrm{min}$, and the injection volume was $2 \mu \mathrm{L}$.

The mass spectrometer was operated in negative ion electrospray ionization mode. The analytes were detected using the multiple-reaction monitoring (MRM) mode with $\mathrm{m} / \mathrm{z}$ transitions at 717.2 $\longrightarrow 519.1$ for Sal B, 179.1 $\longrightarrow 137.0$ for ASA, $136.8 \longrightarrow 65.0$ for $S A$, and $321.0 \longrightarrow 152.0$ for IS. The optimized instrumental conditions were as follows: gas source temperature, $400^{\circ} \mathrm{C}$; ion spray voltage, $4000 \mathrm{~V}$; entrance potential, $10 \mathrm{~V}$; collision cell exit potential, $17 \mathrm{~V}$; curtain gas, 15 psi; gas 1,18 psi; and gas 2, 5 psi. The collision energies and declustering potentials for the analytes are listed in Table 1.

2.3. Preparation of Standard Solutions. To prepare the primary stock solutions $(1 \mathrm{mg} / \mathrm{mL})$, ASA and SA were accurately weighed and dissolved in ACN, whereas Sal B was dissolved in $\mathrm{MeOH}$. The Sal B, ASA, and SA stock solutions were mixed (1: $1: 1, \mathrm{v} / \mathrm{v} / \mathrm{v})$ and serially diluted with $\mathrm{MeOH} / \mathrm{H}_{2} \mathrm{O}(80: 20, \mathrm{v} / \mathrm{v})$ to obtain standard working solutions and quality control (QC) solutions. The IS stock solution was prepared in ACN and diluted with $\mathrm{FA} / \mathrm{H}_{2} \mathrm{O}(50: 50$, v/v) to obtain a working solution at a concentration of $50 \mathrm{ng} / \mathrm{mL}$. All solutions were stored at $4^{\circ} \mathrm{C}$.

2.4. Preparation of Calibration Standards and Quality Control Samples. The calibration standards and QC samples were prepared by spiking $40 \mu \mathrm{L}$ of blank human plasma with $10 \mu \mathrm{L}$ of a working solution. The Sal B, ASA, and SA calibration standards were prepared in plasma at concentrations of 5, 


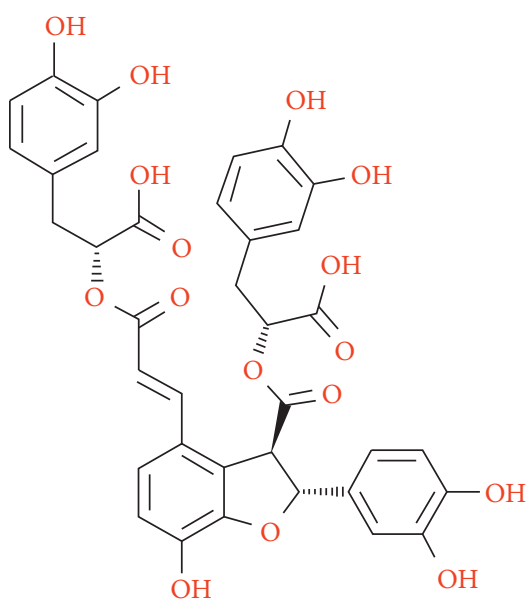

(a)

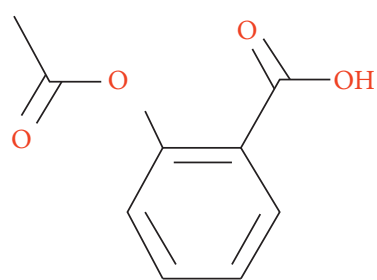

(b)

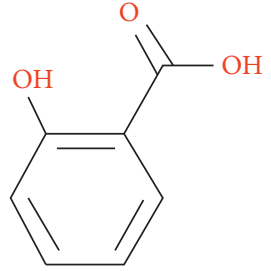

(c)

(c)

(d)

Figure 1: Chemical structures of (a) salvianolic acid B, (b) acetylsalicylic acid, (c) salicylic acid, and (d) chloramphenicol.

TABLE 1: MS/MS ion acquisition parameters of Sal B, ASA, SA, and IS.

\begin{tabular}{lccc}
\hline Components & Transition $(\mathrm{m} / \mathrm{z})$ & Collision energy $(\mathrm{V})$ & Declustering potential $(\mathrm{V})$ \\
\hline Sal B & $717.2 \longrightarrow 519.1$ & -20.0 & -50.0 \\
ASA & $179.1 \longrightarrow 137.0$ & -10.0 & -30.0 \\
SA & $136.8 \longrightarrow 65.0$ & -25.0 & -50.0 \\
IS & $321.0 \longrightarrow 152.0$ & -21.0 & -100.0 \\
\hline
\end{tabular}

10, 50, 300, 800, 2000, 5000, and $6000 \mathrm{ng} / \mathrm{mL}$. The QC samples for Sal B, ASA, and SA were prepared in plasma at four concentrations: $5 \mathrm{ng} / \mathrm{mL}$ for lower limit of quantitation (LLOQ), $15 \mathrm{ng} / \mathrm{mL}$ for low QC (LQC), $3000 \mathrm{ng} / \mathrm{mL}$ for medium QC (MQC), and $4500 \mathrm{ng} / \mathrm{mL}$ for high QC (HQC). The calibration standards and QC samples were freshly prepared every day and stored in an ice-water mixture.

2.5. Sample Preparation. A plasma sample $(50 \mu \mathrm{L})$ spiked with $20 \mu \mathrm{L}$ of IS was vortex-mixed for $30 \mathrm{~s}$. Then, $300 \mu \mathrm{L}$ of ethyl acetate was added to the plasma sample. Liquid-liquid extraction was performed by vortexing the mixture for $2 \mathrm{~min}$ and then centrifuging at $12000 \mathrm{rpm}$ for $10 \mathrm{~min}$ at $4^{\circ} \mathrm{C}$. After separation, the organic layer was evaporated at room temperature under a gentle stream of nitrogen. The residue was redissolved in $50 \mu \mathrm{L}$ of a MeOH/ACN/ $\mathrm{H}_{2} \mathrm{O}$ mixture (40 : $40: 20, \mathrm{v} / \mathrm{v} / \mathrm{v}$ ) and vortex-mixed for $2 \mathrm{~min}$. After centrifugation at $12000 \mathrm{rpm}$ for $10 \mathrm{~min}$ at $4^{\circ} \mathrm{C}, 2 \mu \mathrm{L}$ of the supernatant was analyzed by UPLC-MS/MS.

\subsection{Method Validation}

2.6.1. Specificity. The specificity of the method was investigated using six predose plasma samples from different volunteers. A comparison of the chromatograms of blank plasma from six sources with those of blank plasma spiked with the corresponding analytes confirmed that there was no interference from endogenous compounds at the retention times of the analytes. The quantitative criteria can still be satisfied if the peak areas of endogenous compounds coeluting with the analytes are less than $20 \%$ of the peak area of the LLOQ standard and the IS response in the blank does not exceed $5 \%$ of the average response in the calibration standards and QCs [17].

2.6.2. Calibration Curve Linearity and Method LLOQ. Calibration curves for Sal B, ASA, and SA were established by adding the working solution of the corresponding analyte to blank human plasma at eight different concentrations and performing measurements on three consecutive days. The curves were constructed using the peak area ratios of the analyte to the IS versus the plasma concentration of the analyte with a weighted least-squares regression model. The correlation coefficient $(r)$ was required to be 0.99 or higher. The LLOQ was defined as the minimum concentration on the calibration curve that could be measured with acceptable accuracy (within $\pm 20 \%$ ) and precision (relative standard deviation $(\mathrm{RSD}) \leq 20 \%)$.

2.6.3. Matrix Effect and Extraction Recovery. The matrix effect (ME) was measured by comparing the peak areas of Sal $\mathrm{B}, \mathrm{ASA}, \mathrm{SA}$, and IS in pretreated blank plasma from six sources with those of the neat standard solutions at the same concentration. The ratio between the ME of the analyte and the corresponding ME of IS was defined as the IS-normalized ME with $\mathrm{RSD} \leq 15 \%$. The extraction recovery was calculated using the ratio between the peak area of the analyte in the QC samples and that of the analyte in pretreated blank plasma. Both the recovery and $\mathrm{ME}$ were 
obtained by six replicate measurements at three QC concentrations (LQC, MQC, and HQC).

2.6.4. Precision and Accuracy. The intraday and interday accuracies were evaluated by the determination of the LLOQ, LQC, MQC, and HQC samples with six replicates at each concentration level. The precision was defined as the RSD between the replicate measurements. The accuracy was calculated as the ratio between the observed concentration and the nominal concentration. The accuracy of the QC concentrations should be within $\pm 15 \%$ with RSDs of less than $15 \%$, except for the LLOQ, which requires an accuracy within $\pm 20 \%$ with an RSD of less than $20 \%$ [19].

2.6.5. Stability. The stabilities of Sal B, ASA, and SA were evaluated using three methods, namely, autosampler stability for $24 \mathrm{~h}$, short-term stability for $4 \mathrm{~h}$, and long-term stability at $-80^{\circ} \mathrm{C}$ for 60 days. To determine the autosampler stability, a blank plasma sample spiked with a QC working solution was kept in the autosampler at $4^{\circ} \mathrm{C}$ for $24 \mathrm{~h}$ and then introduced into the UPLC-MS/MS instrument for analysis. The short-term stability was evaluated by storing at room temperature $\left(\sim 25^{\circ} \mathrm{C}\right)$ for $4 \mathrm{~h}$. The long-term stability was determined by storing the spiked plasma samples at $-80^{\circ} \mathrm{C}$ for 60 days. Three QC concentrations (LQC, MQC, and HQC) were investigated for each stability measurement, with six parallel replicates for each concentration.

2.7. Application to a Pharmacokinetic Trial. In the randomized, open-labeled, parallel-grouped, single-center clinical trial, 16 coronary heart disease patients were randomly allocated into three arms. Participants in the aspirin group were given aspirin (100 mg QD), participants in the salvianolate injection group were given a salvianolate injection $(200 \mathrm{mg}+5 \%$ glucose injection, $250 \mathrm{~mL}$, iv), and participants in the combination group were given aspirin (100 mg QD) and salvianolate injection (200 mg + 5\% glucose injection, $250 \mathrm{~mL}$, iv). For each group, the treatment was administered for 10 consecutive days. Blood samples were collected before dosing on days 8,9 , and 10 and at $5 \mathrm{~min}$ (aspirin group only), $15 \mathrm{~min}, 30 \mathrm{~min}, 45 \mathrm{~min}, 1 \mathrm{~h}, 2 \mathrm{~h}$, $4 \mathrm{~h}, 8 \mathrm{~h}, 12 \mathrm{~h}$, and $24 \mathrm{~h}$ after dosing on day 10 . The plasma was separated by centrifugation at $4500 \mathrm{rpm}$ for $10 \mathrm{~min}$ at $4^{\circ} \mathrm{C}$ and then stored at $-80^{\circ} \mathrm{C}$ until analysis. The validated UPLC-MS/MS method was successfully applied to determine the concentrations of Sal B, ASA, and SA in the plasma samples, which allowed the observation of pharmacokinetic changes due to DDIs between salvianolate injection and ASA [20]. The trial was registered on October 9, 2017 (ClinicalTrials.gov, NCT03306550). The pharmacokinetic analysis was performed using the Phoenix WinNonlin 7.0 software (Pharsight, a Certara Company, Mountain View, CA, USA).

\section{Results and Discussion}

3.1. Optimization of the UPLC-MS/MS Conditions. It has been reported that Sal B, ASA, and SA all exhibit higher signal intensities in negative ion mode than in positive ion mode [10-15]. Through Q1 screening, high-intensity and stable peaks were found for the deprotonated molecular ions $[\text { Sal B-H }]^{-},[\text {ASA-H }]^{-},[\mathrm{SA}-\mathrm{H}]^{-}$, and $[\mathrm{IS}-\mathrm{H}]^{-}$. After the precursor ions were further fragmented with medium-intensity collision energy, the two products with the strongest ion signals were selected for each analyte, and the declustering potential and collision energy for each ion pair were optimized separately. Finally, the mass transitions with strong signals were identified $(m / z 717.2 \longrightarrow 519.1$ for Sal B, $m / z$ $179.1 \longrightarrow 137.0$ for ASA, $m / z 136.8 \longrightarrow 65.0$ for SA, and $m / z$ $321.0 \longrightarrow 152.0$ for IS).

ASA generated $[\mathrm{SA}-\mathrm{H}]^{-}$fragment ions during the insource collision-induced dissociation process, so that the chromatographic peak of SA was detected at the retention time of ASA. To avoid affecting the accurate quantification of SA, the ASA and SA chromatographic peaks were separated using an optimized gradient elution method, as described in Section 2.2. As a result, the resolution factor between the two chromatographic peaks was greater than 1.5. Furthermore, lowering the mobile phase $\mathrm{pH}$ significantly improved the tailings of the Sal B and SA chromatographic peaks. In particular, $0.1 \% \mathrm{FA}$ in water and $0.5 \% \mathrm{FA}$ in water were tested as solvent $\mathrm{A}$, and the latter provided better peak shapes than the former.

Because blank plasma contains a certain amount of SA (from food sources) [21], reducing the gas source temperature, ion spray voltage, and SA collision energy improved the baseline. As shown in Figure 2, the signal-to-noise ratios for Sal B, ASA, SA, and IS meet the specificity requirements and can be used for sample analysis.

3.2. Sample Preparation. Liquid-liquid extraction and protein precipitation are the most common methods for plasma processing. Owing to the first-pass effect, the plasma concentration of ASA after oral administration is low [22]. Thus, the use of the organic solvent protein precipitation method would dilute the sample, making the signal strength of the LLOQ insufficient to meet the quantitative requirements. Therefore, we chose the liquid-liquid extraction method to extract the analytes. Generally, liquid-liquid extraction is preferred over protein precipitation because cleaner samples are produced, which may minimize the ME and increase the instrument performance.

When redissolving the nitrogen-dried residue, we found that using an inappropriate combination of solvents led to an unqualified standard curve with $r<0.99$, which may be related to the solubility of the analytes. First, $\mathrm{ACN} / \mathrm{H}_{2} \mathrm{O}$ ratios from $80: 20(\mathrm{v} / \mathrm{v})$ to $20: 80(\mathrm{v} / \mathrm{v})$ were investigated. As the ACN content increased, the ASA and SA curves gradually improved, but most of the concentration points in the Sal B curve remained scattered. When $\mathrm{MeOH}$ was added to the solvent mixture instead of ACN, the standard curve of Sal B became linear. Then, a combination of $\mathrm{MeOH} / \mathrm{H}_{2} \mathrm{O}$ $(80: 20, \mathrm{v} / \mathrm{v})$ was considered, but the ASA curve became nonlinear. Finally, $\mathrm{MeOH} / \mathrm{ACN} / \mathrm{H}_{2} \mathrm{O}(40: 40: 20$, v/v/v) was selected as the solvent mixture to redissolve the nitrogendried residue. 

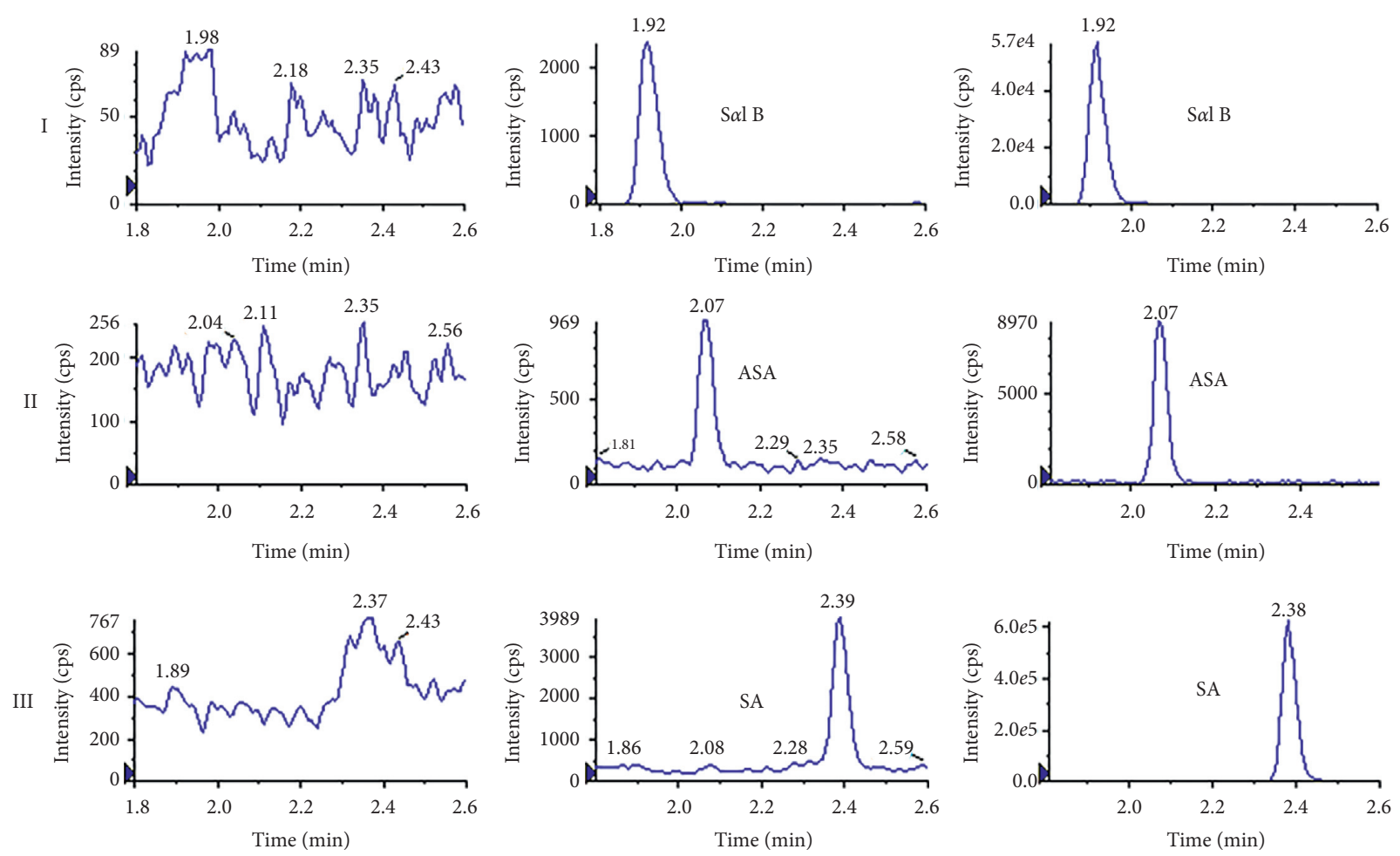

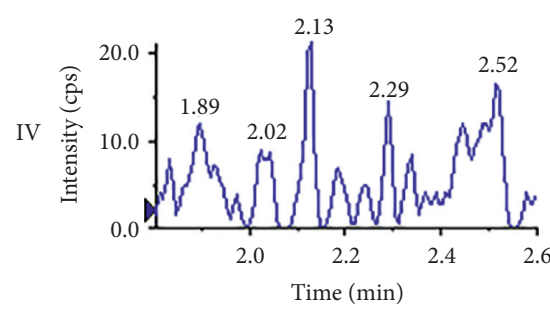

(a)

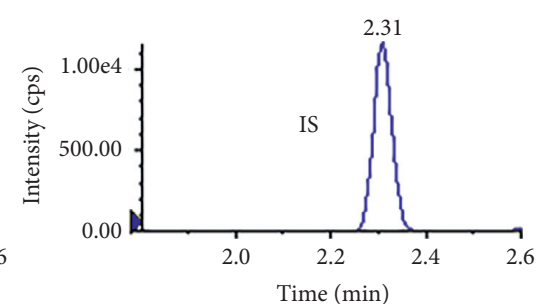

(b)

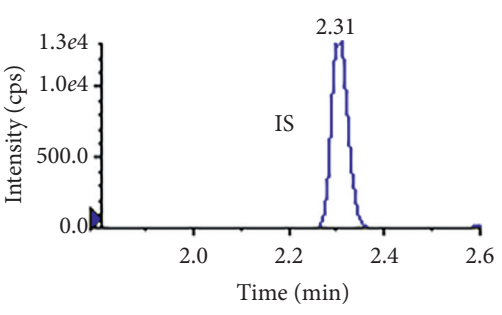

(c)

FIgURe 2: Representative MRM chromatograms of Sal B ASA, SA, and IS in plasma. (a) Blank plasma. (b) LLOQ sample (Sal B ASA, and SA at $5 \mathrm{ng} / \mathrm{mL}$; IS at $20 \mathrm{ng} / \mathrm{mL}$ ). (c) Plasma sample $4 \mathrm{~h}$ after administration.

\subsection{Method Validation}

3.3.1. Selectivity. Typical chromatograms for blank plasma, the LLOQ sample, and an actual plasma sample are shown in Figure 2. The retention times were $1.92 \mathrm{~min}$ for $\mathrm{Sal} \mathrm{B}$, $2.07 \mathrm{~min}$ for ASA, $2.39 \mathrm{~min}$ for SA, and $2.31 \mathrm{~min}$ for IS. These results demonstrated that, under the optimized experimental conditions, no interference peaks were induced by endogenous compounds at the retention times of any of the analytes, and the signal-to-noise ratios of each analyte in the LLOQ samples were greater than 10, which meets the acceptability criteria for quantitative biological analysis.

3.3.2. Calibration Curve Linearity and Sensitivity. Calibration standard curves for Sal B, ASA, and SA in the range 5-6000 ng/mL were established using eight concentrations. The three standard curves for each analyte in Table 2 are the accompanying standard curves for the three batches of precision and accuracy validation. The fitting of the Sal B and ASA data using a least-squares linear model with a weight of $1 / x^{2}$ gave regression correlation coefficients in the range 0.9986-0.9991 for Sal B and 0.9959-0.9972 for ASA. In contrast, the SA data were fitted with a $1 / y$ weighted least-squares quadratic model, which gave regression correlation coefficients in the range 0.9988-0.9999. The LLOQs of Sal B, ASA, and SA met the accuracy requirement within $\pm 20 \%$, and the accuracies of the other concentration points were within $\pm 15 \%$.

SA has a high protein binding rate [23]. Therefore, when $\mathrm{SA}$ is added to blank plasma, some of it binds to plasma proteins, resulting in a low concentration of free SA. Because the amount of albumin in blank plasma is limited, this phenomenon has a much greater effect on the detection of low-concentration SA than on that of high-concentration SA, which is speculated to contribute to the nonlinearity of the SA calibration curve. On the other hand, when the concentration or the ion intensity generated from a target compound exceeds a certain level, mass spectrometers will exhibit a nonlinear response owing to ionization saturation, detector saturation, or both [24]. We found that the SA 
TABLE 2: Calibration curves and correlation coefficients of Sal B, ASA, and SA.

\begin{tabular}{lcc}
\hline Components & Equation & Correlation coefficient $(r)$ \\
\hline \multirow{2}{*}{ Sal B } & $y=0.0499 x+0.0457$ & 0.9991 \\
& $y=0.482 x+0.0697$ & 0.9988 \\
& $y=0.0436 x+0.0595$ & 0.9986 \\
ASA & $y=0.0211 x+0.00794$ & 0.9959 \\
& $y=0.0213 x-0.00818$ & 0.9972 \\
\hline & $y=0.0187 x+0.00661$ & 0.9966 \\
SA & $y=-5.98 e-007 x 2+0.012 x+0.236$ & 0.9999 \\
& $y=-6.67 e-007 x 2+0.013 x+0.277$ & 0.9999 \\
\hline
\end{tabular}

calibration curve was significantly bent at two points near the quantitative upper limit, even if it was constructed using a neat solution with the standard working solutions instead of plasma samples. A similar but less pronounced phenomenon was observed for the ASA curve but not for the Sal B curve. Furthermore, the signal strength of SA was lower than that of ASA or Sal B at the same concentration, which indicated that the nonlinear curve of SA was not attributable to detector saturation and more likely originated from ionization saturation. Thus, at high concentrations, SA was not completely ionized during the ionization process, which could be related to the adjustments made to the mass spectrometry parameters to reduce non-drug-derived SA signals in the plasma samples. However, as shown in Table 2, the regression correlation coefficients of the SA standard curves for the three batches were greater than 0.99; thus, these effects and the nonlinearity of the calibration curve do not affect the efficacy of the developed analytical method.

3.3.3. Extraction Recovery and Matrix Effect. The extraction recovery data indicated that the extraction procedure was efficient for Sal B, ASA, and SA at three QC levels. At concentrations of 15,3000 , and $4500 \mathrm{ng} / \mathrm{mL}$, the average extraction recoveries were $83.3 \pm 4.3 \%, 85.3 \pm 4.3 \%$, and $87.2 \pm 4.9 \%$ for Sal B; $97.9 \pm 5.2 \%, 96.3 \pm 2.6 \%$, and $93.2 \pm 1.8 \%$ for ASA; and $93.1 \pm 4.3 \%, 94.6 \pm 1.3 \%$, and $95.2 \pm 2.1 \%$ for SA. As shown in Table 3, the MEs were in the range $99.7-103 \%$ for Sal $\mathrm{B}, 97.3-102 \%$ for ASA, and $96.7-106 \%$ for $\mathrm{SA}$, which indicated that there were no significant MEs. After normalization of the ME data with the IS, the ME values of each analyte were reduced, which was related to the enhancement of the IS signal by the matrix. The RSDs of the IS-normalized MEs ranged from $3.75 \%$ to $4.55 \%$, which demonstrated that the MEs of the analytes at three concentrations were stable.

3.3.4. Precision and Accuracy. The intraday and interday precisions and accuracies of Sal B, ASA, and SA were determined by analyzing four QC concentrations from three batches with six replicates for each concentration. As shown in Table 4 , the intraday and interday accuracies were within $\pm 8.7 \%$ and $\pm 8.5 \%$ for Sal B, $\pm 4 \%$ and $\pm 5.2 \%$ for ASA, and $\pm 11 \%$ and $\pm 8.9 \%$ for $S A$, respectively. The intraday precisions of Sal B, ASA, and SA were less than $5.31 \%, 6.19 \%$, and $8.52 \%$, respectively, and the interday precisions were less than $7.56 \%, 8.54 \%$, and $9.59 \%$, respectively. The results indicated that the precision and accuracy met the acceptability criteria for biological sample analysis.

3.3.5. Stability. The stability results are summarized in Table 5. QC samples at three concentrations of Sal B, ASA, and SA were stored at room temperature $\left(\sim 25^{\circ} \mathrm{C}\right)$ with an acidifying agent $\left(20 \mu \mathrm{L}\right.$ of $\mathrm{FA} / \mathrm{H}_{2} \mathrm{O}(50: 50, \mathrm{v} / \mathrm{v})$ containing IS) for $4 \mathrm{~h}$ or in a freezer at $-80^{\circ} \mathrm{C}$ for 60 days before analysis. The accuracies of the QC samples were within $\pm 15 \%$, which indicated that the analytes were stable in plasma under these storage conditions. Considering that ASA and SA may be degraded by biological enzymes in plasma, we also investigated the stability of the QC samples at room temperature $\left(\sim 25^{\circ} \mathrm{C}\right)$ without the acidifying agent for $4 \mathrm{~h}$. The results showed that approximately $40 \%$ of ASA was metabolized by plasma esterase to produce SA, demonstrating that acidified plasma inhibits analyte degradation. Therefore, in clinical operation, whole blood should be processed immediately after collection and stored at $-80^{\circ} \mathrm{C}$, and acidifying agents should be added to the thawed plasma as soon as possible to prevent analyte degradation. Furthermore, the QC samples were stored in an autosampler at $4^{\circ} \mathrm{C}$ for $24 \mathrm{~h}$ before analysis. The obtained accuracies also met the acceptability criteria, indicating that the analytes were stable in the autosampler before the analysis was performed.

3.4. Clinical Sample Analysis. The developed method was applied to measure the concentrations of Sal B, ASA, and SA in the plasma samples of 16 patients. Table 6 shows the mean values calculated at each blood collection time point. Most concentrations of Sal B and SA were within the quantitative range, whereas those of ASA were below the LLOQ, which was related to the spacing of the blood collection time points. The pharmacokinetic parameters are shown in Table 7. The $C_{\text {max }}, \mathrm{AUC}_{0-\mathrm{t}}$, and $\mathrm{AUC}_{0-\infty}$ values of the analytes in the combined aspirin and salvianolate injection group $(A+S$ group) were lower than those in the aspirin group (AP group) or the salvianolate injection group (SV group), which indicated lower drug exposure in the $\mathrm{A}+\mathrm{S}$ group. Furthermore, the time required for the SA plasma concentration to reach $C_{\max }$ was different between the $\mathrm{A}+\mathrm{S}$ group and the SV group, suggesting that DDIs changed the absorption or elimination rate of SA. These observations verified that DDIs between aspirin and salvianolate injection affect the pharmacokinetic behavior. 
TABLE 3: Extraction recovery and matrix effects of Sal B, ASA, SA, and IS ( $n=6)$.

\begin{tabular}{lccccc}
\hline Compound & $\begin{array}{c}\text { Nominal conc. (ng/ } \\
\mathrm{mL})\end{array}$ & $\begin{array}{c}\text { Extraction recovery } \\
(\text { mean } \pm \text { SD } \%)\end{array}$ & $\begin{array}{c}\text { ME (mean } \pm \text { SD } \\
\%)\end{array}$ & $\begin{array}{c}\text { IS-normalized ME } \\
(\text { mean } \pm \text { SD } \%)\end{array}$ & $\begin{array}{c}\text { RSD\% (IS-normalized } \\
\text { ME) }\end{array}$ \\
\hline \multirow{3}{*}{ Sal B } & 15 & $83.3 \pm 4.3$ & $101 \pm 4$ & $92.1 \pm 4.3$ & 3.95 \\
& 3000 & $85.3 \pm 4.3$ & $103 \pm 4$ & $94.0 \pm 2.8$ & \\
\hline \multirow{3}{*}{ ASA } & 4500 & $87.2 \pm 4.9$ & $99.7 \pm 4.0$ & $93.3 \pm 3.9$ & \\
& 15 & $97.9 \pm 5.2$ & $102 \pm 3$ & $93.3 \pm 3.1$ & 3.75 \\
\hline & 3000 & $96.3 \pm 2.6$ & $97.3 \pm 3.2$ & $92.3 \pm 1.7$ & \\
SA & 4500 & $93.2 \pm 1.8$ & $101 \pm 2$ & $97.1 \pm 7.0$ & \\
& 15 & $93.1 \pm 4.3$ & $106 \pm 8$ & $95.0 \pm 2.5$ & \\
\hline IS & 3000 & $94.6 \pm 1.3$ & $96.7 \pm 2.6$ & $95.0 \pm 2.6$ & \\
\hline
\end{tabular}

Extraction recovery $=($ mean peak area of analyte in the plasma matrix $) /($ mean peak area of analyte in the extracted plasma matrix $) . M E=($ mean peak area of analyte in the extracted plasma matrix $) /($ mean peak area of analyte in the dilution solution $)$. IS-normalized ME $=(($ mean peak area of analyte in the extracted plasma matrix $) /($ mean peak area of analyte in the dilution solution $)) /(($ mean peak area of IS in the extracted plasma matrix $) /($ mean peak area of IS in the dilution solution $)$ ). $\mathrm{RSD} \%=($ standard deviation $/$ mean $) \times 100 \%$.

TABLE 4: Accuracy and precision of Sal B, ASA, and SA.

\begin{tabular}{|c|c|c|c|c|c|c|}
\hline \multirow[b]{2}{*}{$\begin{array}{l}\text { Nominal conc. (ng/ } \\
\text { mL) }\end{array}$} & \multicolumn{3}{|c|}{ Intraday $(n=6)$} & \multicolumn{3}{|c|}{ Interday $(n=18)$} \\
\hline & $\begin{array}{l}\text { Observed conc. (ng/ } \\
\mathrm{mL} \text { ) }\end{array}$ & $\begin{array}{c}\text { Precision RSD } \\
(\%)\end{array}$ & $\begin{array}{c}\text { Accuracy (RE } \\
\%)\end{array}$ & $\begin{array}{l}\text { Observed conc. (ng/ } \\
\mathrm{mL} \text { ) }\end{array}$ & $\begin{array}{c}\text { Precision RSD } \\
(\%)\end{array}$ & $\begin{array}{c}\text { Accuracy (RE } \\
\%)\end{array}$ \\
\hline \multicolumn{7}{|l|}{ Sal B } \\
\hline 5 & $4.89 \pm 0.26$ & 5.31 & -2.2 & $4.94 \pm 0.37$ & 7.56 & -1.2 \\
\hline 15 & $14.5 \pm 0.5$ & 3.44 & -3.3 & $15.1 \pm 0.8$ & 5.01 & 0.6 \\
\hline 3000 & $2802 \pm 106$ & 3.77 & -6.6 & $2746 \pm 108$ & 3.92 & -8.5 \\
\hline 4500 & $4108 \pm 99$ & 2.42 & -8.7 & $4333 \pm 237$ & 5.46 & -3.7 \\
\hline \multicolumn{7}{|l|}{ ASA } \\
\hline 5 & $4.80 \pm 0.28$ & 5.85 & -4 & $4.96 \pm 0.42$ & 8.54 & -0.8 \\
\hline 15 & $14.8 \pm 0.9$ & 6.19 & -1.3 & $14.4 \pm 0.8$ & 5.48 & -4 \\
\hline 3000 & $3037 \pm 111$ & 3.67 & 1.2 & $3157 \pm 147$ & 4.55 & 5.2 \\
\hline 4500 & $4332 \pm 140$ & 3.22 & -3.7 & $4184 \pm 196$ & 4.70 & -7 \\
\hline \multicolumn{7}{|l|}{ SA } \\
\hline 5 & $5.38 \pm 0.46$ & 8.52 & 7.6 & $5.14 \pm 0.49$ & 9.59 & 2.8 \\
\hline 15 & $15.8 \pm 0.8$ & 5.26 & 5.3 & $15.7 \pm 1.0$ & 6.29 & 4.6 \\
\hline 3000 & $2663 \pm 82$ & 3.09 & -11 & $2734 \pm 134$ & 4.90 & -8.9 \\
\hline 4500 & $4257 \pm 98$ & 2.30 & -5.4 & $4291 \pm 199$ & 4.64 & -4.6 \\
\hline
\end{tabular}

$\mathrm{RSD} \%=($ standard deviation $/$ mean $) \times 100 \%$. RE $\%=($ mean of observed conc. - nominal conc. $) /$ nominal conc. $\times 100 \%$.

TABLE 5: Stability of Sal B, ASA, and SA.

\begin{tabular}{|c|c|c|c|c|c|c|c|c|}
\hline \multirow{2}{*}{$\begin{array}{l}\text { Nominal } \\
\text { conc. }(\mathrm{ng} / \mathrm{mL})\end{array}$} & \multicolumn{2}{|c|}{$\begin{array}{l}\text { Storage in autosampler at } 4^{\circ} \mathrm{C} \\
\text { for } 24 \mathrm{~h}(n=3)\end{array}$} & \multicolumn{2}{|c|}{$\begin{array}{l}\text { Storage in freezer at }-80^{\circ} \mathrm{C} \\
\text { for } 60 \text { days }(n=6)\end{array}$} & \multicolumn{2}{|c|}{$\begin{array}{l}\text { Storage at } 25^{\circ} \mathrm{C} \text { for } 4 \mathrm{~h} \text { after } \\
\text { acidification }(n=6)\end{array}$} & \multicolumn{2}{|c|}{$\begin{array}{c}\text { Storage at } 25^{\circ} \mathrm{C} \text { for } 4 \mathrm{~h} \\
\text { without acidification }(n=6)\end{array}$} \\
\hline & $\begin{array}{c}\text { Observed } \\
\text { conc. }(\mathrm{ng} / \mathrm{mL})\end{array}$ & $\begin{array}{l}\text { Accuracy } \\
\text { (RE\%) }\end{array}$ & $\begin{array}{c}\text { Observed } \\
\text { conc. }(\mathrm{ng} / \mathrm{mL})\end{array}$ & $\begin{array}{l}\text { Accuracy } \\
\text { (RE\%) }\end{array}$ & $\begin{array}{c}\text { Observed } \\
\text { conc. }(\mathrm{ng} / \mathrm{mL})\end{array}$ & $\begin{array}{l}\text { Accuracy } \\
\text { (RE\%) }\end{array}$ & $\begin{array}{c}\text { Observed } \\
\text { conc. }(\mathrm{ng} / \mathrm{mL})\end{array}$ & $\begin{array}{l}\text { Accuracy } \\
\text { (RE\%) }\end{array}$ \\
\hline \multicolumn{9}{|l|}{ Sal B } \\
\hline 15 & $16.7 \pm 0.6$ & 11 & $15.9 \pm 0.8$ & 6 & $15.2 \pm 1.0$ & 1.3 & $10.1 \pm 1.1$ & -33 \\
\hline 3000 & $3047 \pm 85$ & 1.6 & $2920 \pm 63$ & -2.7 & $2767 \pm 85$ & -7.8 & $2580 \pm 61$ & -14 \\
\hline 4500 & $4490 \pm 358$ & -0.2 & $4438 \pm 179$ & -1.4 & $4370 \pm 290$ & -2.9 & $3988 \pm 164$ & -11 \\
\hline \multicolumn{9}{|l|}{ ASA } \\
\hline 15 & $17.0 \pm 0.3$ & 13 & $14.1 \pm 0.5$ & -6 & $15.9 \pm 0.7$ & 6 & $8.47 \pm 0.6$ & -44 \\
\hline 3000 & $3357 \pm 90$ & 12 & $3117 \pm 27$ & 3.9 & $3260 \pm 96$ & 8.7 & $1762 \pm 87$ & -41 \\
\hline 4500 & $4747 \pm 274$ & 5.5 & $4242 \pm 110$ & -5.7 & $4470 \pm 206$ & -0.7 & $2767 \pm 179$ & -39 \\
\hline \multicolumn{9}{|l|}{ SA } \\
\hline 15 & $15.9 \pm 1.3$ & 6 & $14.8 \pm 1.2$ & -1.3 & $16.1 \pm 0.9$ & 7.3 & $18.3 \pm 0.5$ & 22 \\
\hline 3000 & $2797 \pm 59$ & -6.8 & $3138 \pm 86$ & 4.6 & $2892 \pm 37$ & -3.6 & $3767 \pm 78$ & 26 \\
\hline 4500 & $4233 \pm 188$ & -5.9 & $4635 \pm 156$ & 3 & $4293 \pm 91$ & -4.6 & $5738 \pm 216$ & 28 \\
\hline
\end{tabular}

$\mathrm{RE} \%=($ mean of observed conc. - nominal conc. $) /$ nominal conc. $\times 100 \%$. 
TABLE 6: Measured concentrations of Sal B, ASA, and SA in patients.

\begin{tabular}{|c|c|c|c|c|c|c|c|}
\hline \multirow{2}{*}{\multicolumn{2}{|c|}{ Collection time }} & \multicolumn{2}{|c|}{ Sal B conc. (ng/mL) } & \multicolumn{2}{|c|}{ ASA conc. $(\mathrm{ng} / \mathrm{mL})$} & \multicolumn{2}{|c|}{ SA conc. $(\mathrm{ng} / \mathrm{mL})$} \\
\hline & & SV group & $A+S$ group & AP group & $A+S$ group & AP group & $A+S$ group \\
\hline 8th day & Before & $<$ LLOQ & 8.27 & $<$ LLOQ & $<$ LLOQ & 99.8 & 22.8 \\
\hline 9th day & Before & $<$ LLOQ & 7.73 & $<$ LLOQ & $<$ LLOQ & 76.6 & 24.9 \\
\hline \multirow[t]{11}{*}{ 10th day } & Before & $<$ LLOQ & 12.3 & $<$ LLOQ & $<$ LLOQ & 69.7 & 18.5 \\
\hline & $5 \mathrm{~min}$ & NA & NA & $<$ LLOQ & NA & 63.1 & NA \\
\hline & $15 \mathrm{~min}$ & 2078 & 1059 & $<$ LLOQ & $<$ LLOQ & 50.3 & 18.0 \\
\hline & $30 \mathrm{~min}$ & 1274 & 653 & $<$ LLOQ & $<$ LLOQ & 51.8 & 17.3 \\
\hline & $45 \mathrm{~min}$ & 720 & 436 & $<$ LLOQ & $<$ LLOQ & 48.6 & 15.5 \\
\hline & $1 \mathrm{~h}$ & 471 & 246 & $<$ LLOQ & $<$ LLOQ & 46.2 & 18.5 \\
\hline & $2 \mathrm{~h}$ & 211 & 128 & $<$ LLOQ & 12.4 & 27.2 & 375 \\
\hline & $4 \mathrm{~h}$ & 64.5 & 43.3 & 270 & 239 & 1725 & 2157 \\
\hline & $8 \mathrm{~h}$ & 34.0 & 24.9 & 22.9 & 79.2 & 2229 & 1740 \\
\hline & $12 \mathrm{~h}$ & 15.9 & 16.3 & 104 & 6.05 & 1191 & 640 \\
\hline & $24 \mathrm{~h}$ & 14.9 & 9.96 & $<$ LLOQ & $<$ LLOQ & 84.7 & 57.2 \\
\hline
\end{tabular}

NA represents that the administration group was not collected sample at 5 min after administration.

TABLE 7: Mean of pharmacokinetic parameters of Sal B in the SV group and A + S group and SA in AP group and A + S group (mean \pm SD $\%$ ).

\begin{tabular}{lcccc}
\hline Parameter & \multicolumn{2}{c}{ Sal B } & & SA \\
& SV group $(n=5)$ & A + S group $(n=5)$ & AP group $(n=6)$ & A + S group $(n=5)$ \\
\hline$T_{\max }(\mathrm{h})$ & $1.6 \pm 0.1$ & $1.6 \pm 0.1$ & $7.3 \pm 3.0$ & $5.6 \pm 2.2$ \\
$C_{\max }(\mu \mathrm{g} / \mathrm{mL})$ & $2.3 \pm 1.8$ & $1.1 \pm 0.5$ & $3.3 \pm 1.1$ & $2.8 \pm 1.0$ \\
$T_{1 / 2}(\mathrm{~h})$ & $4.4 \pm 1.5$ & $7.3 \pm 5.3$ & $2.6 \pm 0.8$ & $2.8 \pm 0.8$ \\
AUC $_{0-\mathrm{t}}(h \times \mu \mathrm{g} / \mathrm{mL})$ & $3.5 \pm 1.9$ & $1.9 \pm 0.8$ & $24 \pm 7.5$ & $19 \pm 8.9$ \\
AUC $_{0-\infty}(h \times \mu \mathrm{g} / \mathrm{mL})$ & $3.6 \pm 2.0$ & $2.1 \pm 0.9$ & $24 \pm 7.3$ & $20 \pm 9.0$ \\
\hline
\end{tabular}

$T_{\max }$ : time point of maximum plasma concentration; $C_{\max }$ : maximum plasma concentration; $T_{1 / 2}$ : half-life of drug elimination during the terminal phase; $\mathrm{AUC}_{0-\mathrm{t}}$ : area under the plasma concentration-time curve from zero hours to last time point; and $\mathrm{AUC}_{0-\infty}$ : area under the plasma concentration-time curve from zero hours to infinity.

\section{Conclusions}

A fast, simple, and stable UPLC-MS-/MS method for the simultaneous quantification of Sal B, ASA, and SA in human plasma was developed and validated. The analytes were extracted from acidified plasma by liquid-liquid extraction, separated by gradient elution on a C18 column, and quantified using MRM with negative ion electrospray ionization. The method was successfully applied to the determination of Sal B, ASA, and SA concentrations in human plasma samples from 16 patients to observe the pharmacokinetic changes caused by DDIs between salvianolate injection and ASA. This method is expected to be applicable to follow-up studies in this area as well as other clinical studies of aspirin combined with Chinese medicine preparations containing a Sal B component. Furthermore, this method provides a reference for the development of in vivo quantitative analysis methods for similar compounds to further assist with pharmacokinetic research on the combined application of Chinese and Western medicines.

\section{Data Availability}

The research data used to support the findings of this study are included within the Supplementary Materials file.

\section{Conflicts of Interest}

The authors declare that there are no conflicts of interest regarding the publication of this paper.

\section{Acknowledgments}

The authors thank Editage (http://www.editage.cn) for English language editing. This work was supported by the National Natural Science Foundation of China (81603505); the China Academy of Chinese Medical Sciences (no. zz0908022); the National Key Research and Development Project (2018YFC1706006); and the National Science and Technology Major Project (2017ZX09304003).

\section{Supplementary Materials}

Supplementary material associated with this report contains the data for method validation, which can be found online. (Supplementary Materials)

\section{References}

[1] J. Ren, L. Fu, S. H. Nile, J. Zhang, and G. Kai, "Salvia miltiorrhiza in treating cardiovascular diseases: a review on its 
pharmacological and clinical applications," Frontiers in Pharmacology, vol. 10, p. 753, 2019.

[2] D. Zhang, J. Wu, S. Liu, X. Zhang, and B. Zhang, "Salvianolate injection in the treatment of unstable angina pectoris: a systematic review and meta-analysis," Medicine, vol. 95, no. 51, p. e5692, 2016.

[3] P. Dong, H. Hu, X. Guan et al., "Cost-consequence analysis of salvianolate injection for the treatment of coronary heart disease," Chinese Medicine, vol. 13, p. 28, 2018.

[4] H. Qiu, W. Liu, T. Lan et al., "Salvianolate reduces atrial fibrillation through suppressing atrial interstitial fibrosis by inhibiting TGF- $\beta 1 / \mathrm{Smad} 2 / 3$ and TXNIP/NLRP3 inflammasome signaling pathways in post-MI rats," Phytomedicine, vol. 51, pp. 255-265, 2018.

[5] L. Pan, J. Li, Y. Zhang et al., "Salvianolic acid B inhibits platelets as a P2Y12 antagonist and PDE inhibitor: evidence from clinic to laboratory," Thrombosis Research, vol. 134, no. 4, pp. 866-876, 2014.

[6] S. Zhang, A. Zhong, X. Bu et al., "Salvianolic acid B inhibits platelets-mediated inflammatory response in vascular endothelial cells," Thrombosis Research, vol. 135, no. 1, pp. 137-145, 2015.

[7] F.-f. Ma, Y.-F. Liu, Y. Liu, and Y.-X. Zhao, "Sulfotanshinone sodium injection could decrease fibrinogen level and improve clinical outcomes in patients with unstable angina pectoris," International Journal of Cardiology, vol. 135, no. 2, pp. 254-255, 2009.

[8] J.-p. Li, J.-m. Guo, Y.-q. Hua et al., "The mixture of Salvia miltiorrhiza-Carthamus tinctorius (Danhong injection) alleviates low-dose aspirin induced gastric mucosal damage in rats," Phytomedicine, vol. 23, no. 6, pp. 662-671, 2016.

[9] L. Zhu, S. Wang, Z. Zhang et al., "Pharmacokinetic and pharmacodynamic interaction of Danshen-Gegen extract with warfarin and aspirin," Journal of Ethnopharmacology, vol. 143, no. 2, pp. 648-655, 2012.

[10] M. San Lau, C. Qian, X. Luo et al., "Impact of the Chinese herbal medicines on dual antiplatelet therapy with clopidogrel and aspirin: pharmacokinetics and pharmacodynamics outcomes and related mechanisms in rats," Journal of Ethnopharmacology, vol. 235, pp. 100-110, 2019.

[11] Y. S. Yang, C. P. Pandey, H. Chandasana et al., "Simultaneous quantitation of acetylsalicylic acid and clopidogrel along with their metabolites in human plasma using liquid chromatography tandem mass spectrometry," Biomedical Chromatography, vol. 30, no. 3, pp. 466-473, 2016.

[12] S. R. Laxman, N. R. Pilli, and V. Gandu, "High performance liquid chromatography mass spectrometric method for the simultaneous quantification of pravastatin and aspirin in human plasma: pharmacokinetic application," Journal of Pharmaceutical Analysis, vol. 2, no. 3, pp. 206-213, 2012.

[13] R. Gajula, N. R. Pilli, V. B. Ravi et al., "Simultaneous determination of atorvastatin and aspirin in human plasma by LC-MS/MS: its pharmacokinetic application," Scientia Pharmaceutica, vol. 80, no. 4, pp. 923-940, 2012.

[14] D. Zhao, Z.-d. Gao, D.-e. Han et al., "Influence of rifampicin on the pharmacokinetics of salvianolic acid B may involve inhibition of organic anion transporting polypeptide (Oatp) mediated influx," Phytotherapy Research, vol. 26, no. 1, pp. 118-121, 2012.

[15] P. Li, Y. Xing, Z. Xue et al., "Pharmacokinetics of salvianolic acid $\mathrm{B}$, rosmarinic acid and Danshensu in rat after pulmonary administration of Salvia miltiorrhiza polyphenolic acid solution," Biomedical Chromatography, vol. 33, no. 8, p. e4561, 2019.
[16] S.-M. Li, Z.-H. Yang, and X.-B. Sun, "Simultaneous determination of six Salvia miltiorrhiza gradients in rat plasma and brain by LC-MS/MS," Zhongguo Zhong Yao Za Zhi, vol. 39, no. 9, pp. 1704-1708, 2014.

[17] Y.-T. Wu, Y.-F. Chen, Y.-J. Hsieh, M.-S. Shiao, and T.-H. Tsai, "Bioavailability of salvianolic acid B in conscious and freely moving rats," International Journal of Pharmaceutics, vol. 326, no. 1-2, pp. 25-31, 2006.

[18] R. Jaw, K. Sharma, and N. R. Srinivas, "Review of HPLC methods and HPLC methods with mass spectrometric detection for direct determination of aspirin with its metabolite(s) in various biological matrices," Biomedical Chromatography: BMC, vol. 26, no. 8, pp. 906-941, 2012.

[19] Food and Drug Administration, Bioanalytical Method Validation: Guidance for Industry, Food and Drug Administration, Silver Spring, MA, USA, 2018.

[20] W. Cao, Q. Yang, W. Zhang et al., "Drug-drug interactions between salvianolate injection and aspirin based on their metabolic enzymes," Biomedicine \& Pharmacotherapy, vol. 135, p. 111203, 2021.

[21] G. G. Xu and A. D. Wood, "Natural salicylates: foods, functions and disease prevention," Food \& Function, vol. 2, no. 9, pp. 515-520, 2011.

[22] P. F. Haastrup, T. Grønlykke, and D. E. Jarbøl, "Enteric coating can lead to reduced antiplatelet effect of low-dose acetylsalicylic acid," Basic \& Clinical Pharmacology \& Toxicology, vol. 116, no. 3, pp. 212-215, 2015.

[23] W. Li, J. Zhang, and F. L. S. Tse, Handbook of LC-MS Bioanalysis: Best Practices, Experimental Protocols, and Regulations, John Wiley \& Sons, Hoboken, NY, USA, 2013.

[24] G. Liu, Q. C. Ji, and M. E. Arnold, "Identifying, evaluating, and controlling bioanalytical risks resulting from nonuniform matrix ion suppression/enhancement and nonlinear liquid Chromatography-Mass spectrometry assay response," Analytical Chemistry, vol. 82, no. 23, pp. 9671-9677, 2010. 